\title{
Feasibility and efficacy of minimally invasive stand-alone surgical ablation of atrial fibrillation. A single-center experience
}

\author{
Massimo Santini • Vincenzo Loiaconi • \\ Maria Pia Tocco • Francesco Mele • Claudio Pandozi
}

Received: 31 March 2011 /Accepted: 19 September 2011 / Published online: 11 January 2012

(C) The Author(s) 2012. This article is published with open access at Springerlink.com

\begin{abstract}
Purpose Minimally invasive surgical ablation for atrial fibrillation (AF) has shown good results and low complications incidence. Our objective was to evaluate feasibility and efficacy of this technique in our center.

Methods The procedure included pulmonary vein isolation, ganglionic plexi ablation, ligament of Marshall resection, and left atrial appendage exclusion through beating heart minimally invasive bilateral thoracotomies. Patients were monitored daily by telemedicine during the first 4 months and then by quarterly $24-\mathrm{h}$ Holter monitoring or by implantable cardiac monitor. Ablation success was defined as freedom from any atrial tachyarrhythmia recurrence lasting more than $30 \mathrm{~s}$ and from antiarrhythmic drugs. All patients were followed up for a minimum of 12 months.

Results Twenty-two consecutive patients with AF, paroxysmal in $27 \%$ and persistent in $73 \%$, were treated. Mean age was $63 \pm 10$ years, $86 \%$ were men. Seventy-three percent of patients had previously undergone to one or more catheter ablations. Median follow-up period was 22 months $\left(25^{\circ}-75^{\circ}\right.$ percentile, 20-27). Patients free from any arrhythmia recurrence for at least 6 consecutive months discontinued antiarrhythmic therapy. Ablation was successful in $73 \%$ of patients at 12 months. Freedom from AF recurrences independently from antiarrhythmic therapy status was $91 \%$ at 12 months. Results were consistent in patients that reached 24 months follow-up. There were no deaths. Complications were: one conversion to sternotomy owing to thoracic adherences, one pacemaker implant, and one postoperative hemothorax requiring surgical revision.
\end{abstract}

M. Santini $(\bowtie) \cdot$ V. Loiaconi $\cdot$ M. P. Tocco $\cdot$ F. Mele $\cdot$ C. Pandozi Department of Cardiology, S. Filippo Neri Hospital,

Via Martinotti 20,

00135 Rome, Italy

e-mail: m.santini@rmnet.it
Conclusions Our results show that minimally invasive surgical ablation was feasible and gave satisfactory results at long-term term follow-up in patients with AF.

Keywords Minimally invasive stand-alone surgical ablation · Atrial fibrillation · Pulmonary vein isolation

\section{Introduction}

Atrial fibrillation is the most common sustained cardiac arrhythmia, affecting around $1 \%$ of the global population [1]. Symptoms are caused by a poorly controlled or irregular ventricular rate, and the associated risk of death is doubled in patients with a history of atrial fibrillation [2]. In recent years, transvenous pulmonary vein isolation has become the first choice for patients with highly symptomatic atrial fibrillation refractory to antiarrhythmic drugs [3]. The reported percentage of success of this procedure varies from $45 \%$ to $85 \%$ according to patients' characteristics, adopted technique, and ECG monitoring method used during followup [4-6]. Transvenous pulmonary vein isolation is not exempt from major complications and, in one third of patients, more than one procedure is necessary [7].

More recently, various techniques of minimally invasive surgical pulmonary vein isolation have been developed, which have yielded success rates of $50-100 \%$, depending on primary endpoint definition and ECG monitoring method, with an incidence of major complications inferior to $5 \%$ [8-14]. The European Society of Cardiology suggests considering stand-alone surgical ablation in patients with symptomatic atrial fibrillation refractory to antiarrhythmic drugs that prefer a surgical approach, have undergone one or more failed attempts at catheter ablation, or are not candidates for catheter ablation [15]. We describe 
our experience with minimally invasive stand-alone surgical ablation in patients with atrial fibrillation.

\section{Methods}

\subsection{Patient population}

From June 2008 to May 2010, 22 patients ( $86 \%$ male, mean age $60 \pm 11$ ) underwent stand-alone surgical ablation of atrial fibrillation in our center. These patients had highly symptomatic idiopathic atrial fibrillation refractory to at least two antiarrhythmic drugs: paroxysmal in $6(23 \%)$ and persistent in $16(73 \%)$ according to the definitions of the ESC 2010 Guidelines [16]. The mean ejection fraction in our population was $61 \pm 12 \%$. Idiopathic dilated cardiomyopathy was present in only one patient with depressed ejection fraction $(<35 \%)$ and a previously implanted ICD. Sixteen patients $(73 \%)$ were affected by hypertension but with no structural cardiac involvement. CHADS2 score was 1 for all the patients except for one that had a previous transient ischemic attack. Fifteen patients $(68 \%)$ had previously undergone a mean of $2.6 \pm 2.8$ electrical cardioversions, $6(27 \%)$ at least one pharmacological cardioversion, and $16(73 \%)$ a mean of $1.6 \pm 1.1$ catheter pulmonary vein ablations. Six patients, two with paroxysmal and four with persistent atrial fibrillation, opted for minimally invasive surgical ablation as first procedure, without undergoing previous catheter ablation. Table 1 summarizes the baseline characteristics of the patients.

\subsection{Surgical procedure}

The procedure includes pulmonary vein isolation, ganglionic plexi ablation, ligament of Marshall resection, and left atrial appendage exclusion by means of beating heart minimally invasive bilateral thoracotomies. Oral anticoagulation is discontinued 3 days before the procedure and replaced by heparin that is suspended $12 \mathrm{~h}$ before surgery. All the patients undergo to transesophageal echocardiography before the procedure.

Patients receive general anesthesia and are intubated with a double-lumen tube (Carlens). The operation starts at the right side of the chest. On the operating table, the patient is placed in the classic lateral position on the left side and a 5-6.0-cm incision is made in the third or fourth right intercostal space; a small incision is also made on the midaxillary line in the sixth intercostal space for the camera port (Fig. 1). The subcutaneous tissue and the intercostal musculature are dissected while maintaining the anatomical integrity of the intercostal muscles, in order to avoid bleeding and hematomas, and a soft tissue retractor and a small rib spreader are inserted.
Table 1 Baseline characteristics of the patients

\begin{tabular}{ll}
\hline & $N=22$ \\
\hline Age (years) & $63 \pm 10$ \\
Male, $n$ (\%) & $19(86)$ \\
Atrial fibrillation type & \\
Paroxysmal, $n$ (\%) & $6(27)$ \\
Persistent, $n$ (\%) & $16(73)$ \\
LAD (mm) & $47 \pm 5$ \\
LVEF (\%) & $61 \pm 12$ \\
Hypertension & $16(73 \%)$ \\
Diabetes & $1(5 \%)$ \\
Idiopathic dilated cardiopathy & $1(5 \%)$ \\
Previous ICD implanted & $1(5 \%)$ \\
Previous TIA/stroke & $1(5 \%)$ \\
Oral anticoagulation & $20(91 \%)$ \\
Amiodarone & $13(59 \%)$ \\
Flecainide or propafenone & $13(59 \%)$ \\
Sotalol & $4(18 \%)$ \\
Ca-antagonist & $2(9 \%)$ \\
\hline
\end{tabular}

$I C D$ implantable cardiac defibrillator, $L A D$ left atrial diameter, $L V E F$ left ventricular ejection fraction, TIA transient ischemic attack

Under thoracoscopy, the phrenic nerve is identified and the pericardium is opened $2 \mathrm{~cm}$ in front of the nerve, the superior vena cava, and inferior vena cava to display the heart (Fig. 1). Stay sutures are then placed for better exposure of pulmonary veins and to facilitate dissection in transverse and oblique sinus. Blunt dissection is performed on the upper part of the pulmonary veins and also in oblique sinus in front of inferior vena cava. Pulmonary veins are completely encircled by means of a special dissector (Navigator;

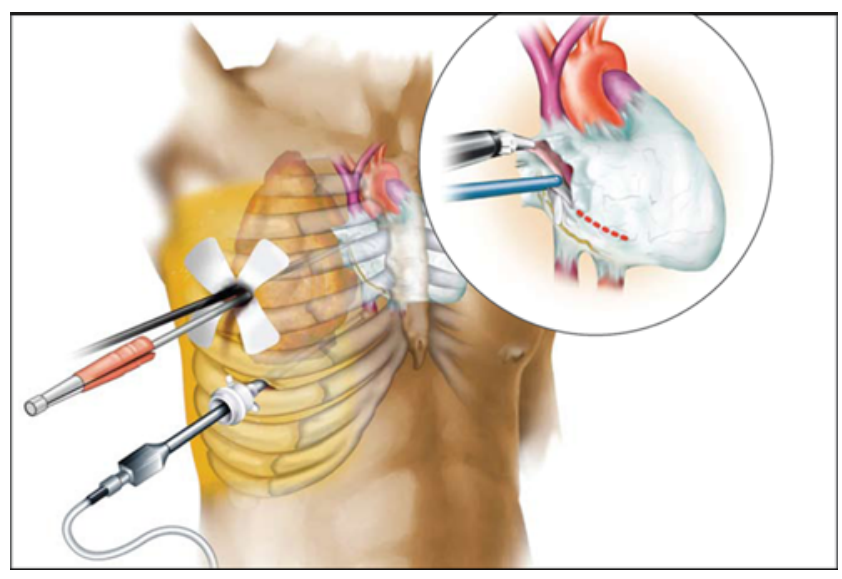

Fig. 1 Surgical procedure. A 5-6.0-cm incision is made in the third or fourth right intercostal space; a small incision is also made on the midaxillary line in the sixth intercostal space for the camera port. The pericardium is opened $1-2 \mathrm{~cm}$ anterior to the phrenic nerve (this figure has been kindly provided by Medtronic Inc.) 
Medtronic, USA). A rubber band is placed around the right pulmonary veins.

To identify predominantly vagal ganglionic plexi, highfrequency electrical stimulation (800 pulses per minute, pulse width $9.9 \mathrm{~ms}$ ) was applied at 10 sites on the epicardial fat pads of the left atrium and pulmonary veins according to a map adapted from Warren Jackman, University of Oklahoma [10], by using an electrosurgical irrigated, monopolar, radiofrequency ablation device (MAPS, Medtronic Inc.). MAPS device is able to map, ablate, pace, and sense in bipolar mode. When stimulated, a transient AV block is induced due to vagal response and the correlating autonomic ganglia are identified. Positive response was defined as $\geq 50 \%$ increase in mean $R-R$ interval [10]. The regions with autonomic ganglia, and also the whole of the para-atrial fat tissue, are then ablated by means of irrigated radiofrequency energy, which is delivered by the same device. Ablation is carried out at a power of $20-25 \mathrm{~W}$. The endpoint for autonomic ganglia ablation is the nonreproducibility of the transient AV block.

The bipolar radiofrequency clamp (Gemini-S, Medtronic Inc.) is then inserted, and two transmural lines are created around the pulmonary veins: one in the direction of the right lung and the other in the direction of the right atrium. The radiofrequency generator emits an acoustic and a visual signal when transmurality is achieved. In order to test the electrical isolation of the pulmonary veins, sensing and pacing no less than $1 \mathrm{~cm}$ away from the visible lesion are carried out by the MAPS device. The endpoint for pulmonary vein ablation is their complete electrical isolation in terms of entrance and exit block into and from them. Entrance block assessment is accomplished by monitoring atrial EGM potentials pre- and postablation on both sides of the lesion for comparison to each other and to those measured previously. Conduction block is demonstrated by absence or reduced potentials in the area isolated by the ablation lesion when compared to left atrial activity outside that area. In patients in sinus rhythm during the procedure, a pacing of the isolated area is performed to verify the presence of conduction block; in patients in atrial fibrillation during the procedure, the sensing is performed inside the isolated area to verify the presence of a significant drop in its electrical activity when compared to others (Fig. 2). The ablation is repeated until the complete electrical isolation is demonstrated.

The right side procedure is completed by inserting a chest tube (16-Fr Blake drain) through the incision of the camera port. The wound is closed in the standard manner with one or two strong prolene pericostal sutures, and subcutaneous and intracutaneous skin sutures.

The patient is then turned into the right lateral position and exactly the same procedure is repeated, the only difference being that the pericardium is opened $2 \mathrm{~cm}$ below the phrenic nerve and the Marshall ligament is separated by means of sharp dissection and ablated with the MAPS device. Autonomic ganglia are then mapped and ablated as described above. The bipolar radiofrequency clamp is led around the left pulmonary veins with the greater curve of
Fig. 2 Example of pre- and postablation sensing performed in patient in atrial fibrillation during the procedure to verify the conduction block. The EGM traces inside and outside the isolated area are compared before and after the ablation. Trace from inside isolated area postablation (c) can be either silent or in its own rhythm if the ectopic foci happen to be firing. In either case, this should look very different from the trace taken from same area preablation (a) and from outside the isolated area preand postablation (b and $\mathbf{d}$, respectively) due to the decreased potentials
(A)

Sensing near PV (area to be isolated)

(B) Sensing on atrium (area not to be isolated)

\section{Pre-ablation}
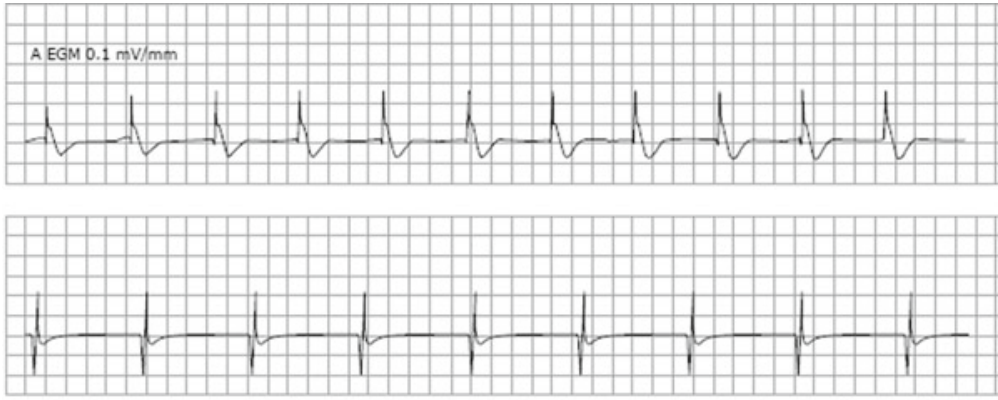

\section{Post-ablation}

(C)

Sensing inside the isolated area

(D)

Sensing on atrium outside the isolated area
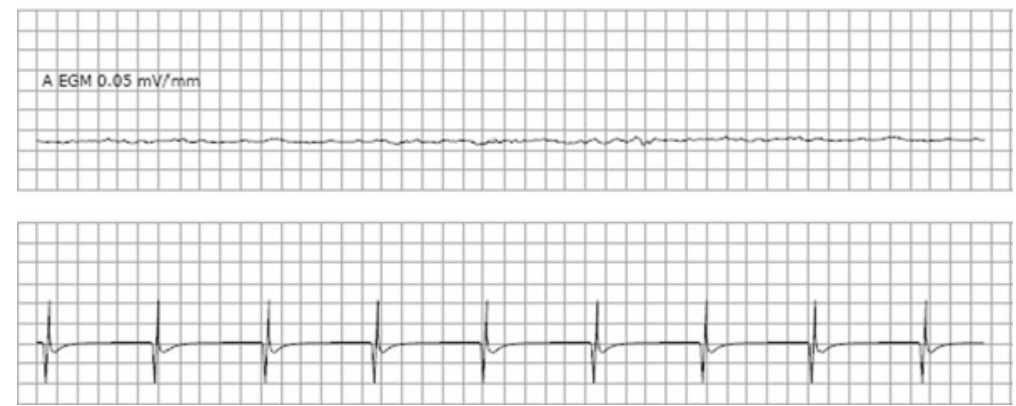
the clamp directed to the left atrium. The clamp is closed at the atrial cuff of the connection of the pulmonary veins to the left atrium, and two ablation lines are created at a distance of 4-5 $\mathrm{mm}$ from each other.

Again, the effectiveness of the block is checked by the MAPS device. The left atrial appendage is then excluded by means of a purse-string suture around the base, using a Teflon pledget-supported $4-0$ prolene suture. The left side procedure is also completed by inserting a drainage tube and closing the wound. At the end of the procedure, $300 \mathrm{mg}$ of amiodarone is administered intravenously to all patients and, if the arrhythmia persists, electrical cardioversion is performed.

\subsection{Postoperative and follow-up management}

After surgery, the patients were transferred to the intensive care unit for $24 \mathrm{~h}$ observation. Chest X-ray was performed routinely in the intensive care unit. Postablation heart monitoring was performed by telemetry and 48-h Holter recording. Drainage tubes were usually removed during the first postoperative day. Amiodarone was administered intravenously to all patients during the first day $(900 \mathrm{mg} / 24 \mathrm{~h})$. In the event of persistent atrial fibrillation recurrence, electrical cardioversion was performed after sedation. All patients received the following drug therapy after surgery: $600 \mathrm{mg} /$ day of amiodarone during the first postoperative week, $400 \mathrm{mg} /$ day of amiodarone during the second postoperative week, and $200 \mathrm{mg}$ /day of amiodarone from the third postoperative week onwards. If the patient was intolerant to amiodarone, $150 \mathrm{mg}$ /day of flecainide or $450 \mathrm{mg} /$ day of propafenone associated to $80 \mathrm{mg} /$ day of sotalol was prescribed.

If the patient had no any arrhythmia recurrence for at least 6 consecutive months of follow-up, the antiarrhythmic therapy was discontinued. Except for the only patient with high risk factor (CHADS2 score=3), oral anticoagulation therapy was prescribed on discharge only to patients with arrhythmia recurrence during the hospital stay.

The first 19 treated patients were enrolled in a telemedicine program during the first 4 months of follow-up and were instructed to transmit $3 \mathrm{~min}$ of ECG four times per day and in case of symptoms. The last three treated patients received an implantable cardiac monitor (Reveal XT, Medtronic Inc.) on the same day of the surgical procedure. All the patients performed an ambulatory examination after $3,6,9$, and 12 months from the procedure and then every 6 months. Patients with no cardiac monitor implanted performed a 24-h Holter after the end of the telemedicine program at 6,9, and 12 months and then every 6 months. Patients were also instructed to come to the hospital in case of symptoms for extra ECG recording. At the 6-month follow-up examination, echocardiography was performed in all patients.

\subsection{Study endpoints}

Freedom from any atrial arrhythmia recurrence lasting more than $30 \mathrm{~s}$ at 12 months follow-up, off antiarrhythmic drugs and regardless of presence or absence of symptoms, was deemed to indicate procedure success. Secondary endpoints considered were freedom from any atrial arrhythmia recurrence lasting more than $30 \mathrm{~s}$ and freedom from atrial fibrillation recurrence lasting more than $30 \mathrm{~s}$ independently from the antiarrhythmic therapy status.

\subsection{Statistical analysis}

Continuous variables were expressed as means and standard deviations, if normal, and as median and range, if not normal. Skewness was assessed by means of Shapiro-Wilk test. Discrete variables were expressed as absolute and relative frequencies. All analyses were carried out by means of the SPSS 12.02 statistical package (SPSS Inc, Chicago, IL).

\section{Results}

\subsection{Perioperative results}

The surgical procedure, including autonomic ganglia ablation, ligament of Marshall resection, and left atrial appendage exclusion, was successfully completed in all patients. The median length of the surgical procedure was $3.2 \mathrm{~h}$ (range 2.1 to $5.5 \mathrm{~h}$ ) from the time of initial skin incision to skin closure on the contralateral side. At the beginning of the procedure, 18 patients $(82 \%)$ were in atrial fibrillation. In patients that previously performed catheter ablation, adherences due to atrial injury were found that made more difficult to isolate pulmonary vein; anyway, this was successfully performed in all the patients.

Three patients had operative complications: one conversion to sternotomy due to thoracic adherences, one need for permanent pacemaker, and one postoperative hemothorax requiring surgical revision. In three patients, intraoperative cardioversion was necessary in order to achieve sinus rhythm. The mean duration of postoperative stay in the intensive care unit was $2.1 \pm 2.2$ days. Four patients $(18 \%)$ had atrial fibrillation recurrences before discharge and required electrical cardioversion; in one case, this was unsuccessful. The mean total hospital stay was $4.3 \pm$ 2.1 days. At the time of discharge, 21 patients (95\%) were in sinus rhythm. 


\subsection{Follow-up results}

The median duration of follow-up was 22 months $\left(25^{\circ}-75^{\circ}\right.$ percentile, 20-27 months). Ablation success in terms of freedom from any atrial arrhythmia recurrence (atrial fibrillation/flutter/tachycardia) lasting more than $30 \mathrm{~s}$ and antiarrhythmic drugs off was reached in $73 \%$ of patients at 12 months (Fig. 3). Freedom from any arrhythmia recurrences independently from antiarrhythmic therapy status was $77 \%$, while freedom from atrial fibrillation recurrences only was $91 \%$ at 12 months (Fig. 3). These results were consistent in the 10 patients that reached the 24-month follow-up.

Figure 4 reports the diagram of arrhythmia recurrences and management. Fourteen patients $(64 \%)$ never had any atrial arrhythmia recurrences and could discontinue the antiarrhythmic drug after the 6-month follow-up visit. One patient always remained in atrial fibrillation, and it was decided to not further treat him due to his previous transient ischemic attack. Other three patients had atrial fibrillation recurrences after an average time of $3 \pm 3$ months, while four had new-onset persistent left atrial flutter episodes after an average time of $8 \pm 5$ months following surgical ablation.

Table 2 summarizes the detailed treatments and outcome of the patients with at least one atrial arrhythmia recurrence. The four patients $(18 \%)$ with persistent left atrial flutter recurrences were indicated to flutter ablation (cavotricuspid isthmus ablation plus linear lesion between the mitral annulus and the superior right pulmonary vein): two patients already performed it; one is waiting for it, while another one refused to perform it. Flutter ablation was successful in one of the two patients while the other one reported atrial tachycardia episodes after the procedure.

One of the two patients that reported paroxysmal atrial fibrillation recurrences underwent thyroidectomy to treat multinodular goiter caused by amiodarone intoxication. The other one only had only one episode $<24 \mathrm{~h}$ at 15 days from the surgical procedure that terminated spontaneously. At the 9-month follow-up, both patients that reported paroxysmal atrial fibrillation recurrences were in sinus rhythm from at least 6 month and could discontinue antiarrhythmic therapy. Another patient always reported persistent atrial fibrillation recurrences in spite of a successful electrical cardioversion.

When patients were grouped according to the type of atrial fibrillation recorded at the baseline, similar results were found: at the 12-month follow-up, $67 \%$ of patients with history of paroxysmal atrial fibrillation were free from atrial arrhythmia recurrences and from antiarrhythmic drugs, as against $75 \%$ of patients with a history of persistent atrial fibrillation. Anyway our population is too small to perform a significant comparison.

A previous performed catheter ablation was not predictive a better outcome. All the patients with early arrhythmia recurrence before discharge had recurrences during follow-up. Mean ejection fraction at 6 months follow-up was similar to the baseline one $(61 \% \pm 11 \%)$. No major cardiac complications, both related and not related to the procedure, were reported during follow-up.

\subsection{Medications}

Oral anticoagulation therapy was prescribed on discharge only to the five patients (23\%) who had had atrial fibrillation episodes during the postoperative period, including the only patient with high risk factor (CHADS2 score $=3$ ). Owing to atrial arrhythmia recurrence during follow-up, another three patients restarted oral anticoagulation therapy during the follow-up. A total of seven patients $(32 \%)$ were taking oral anticoagulation therapy at the last follow-up examination.

Antiarrhythmic therapy was completely discontinued in $16(\%)$ patients at the 12-month follow-up examination, as
Fig. 3 Primary endpoint: percentage of patients free from any atrial arrhythmia recurrence and from antiarrhythmic drugs at difference follow-up. Secondary endpoints: percentage of patients free from any atrial arrhythmia recurrence and percentage of patients free from atrial fibrillation recurrence. $A F$ atrial fibrillation, $A A D$ antiarrhythmic drugs






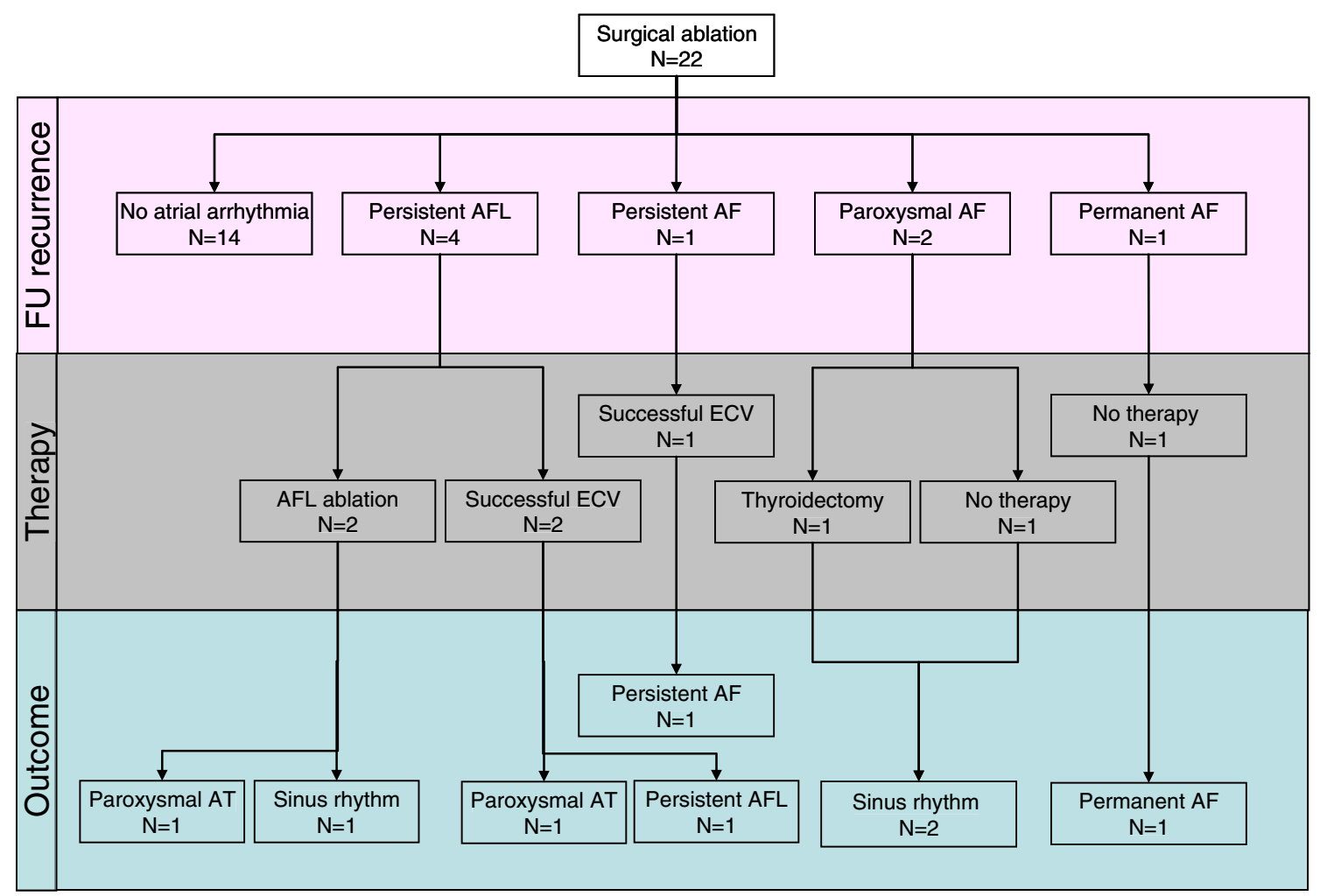

AF: atrial fibrillation. AFL: atrial flutter. AT: atrial tachycardia. ECV: electrical cardioversion. FU: follow-up.

Fig. 4 Arrhythmia recurrences and management diagram. $A F$ atrial fibrillation, $A F L$ atrial flutter, $A T$ atrial tachycardia, $E C V$ electrical cardioversion, $F U$ follow-up

no arrhythmia recurrence had been recorded for at least the last 6 months of follow-up. The patient that performed successful flutter ablation is still under antiarrhythmic therapy since he is in sinus rhythm for only 5 months.

\section{Discussion}

This paper describes our single-center experience with a minimally invasive stand-alone surgical ablation technique that combines pulmonary vein isolation, targeted partial autonomic denervation, and left atrial appendage exclusion. This new technique was developed in 2008 by Bauer [11], and in the last 2 years, many authors have reported good results in terms of safety and freedom from atrial fibrillation on medium-term follow-up, after using different devices and methodologies [8-14]. Owing to the possibility of creating transmural lesions on the beating heart through alternative, less invasive incisions, surgical ablation is gaining ground in patients with atrial fibrillation. It is now also becoming general practice to treat atrial fibrillation in patients that are candidates for major surgery, such as mitral valve replacement/repair or coronary artery bypass graft, since this arrhythmia is an independent risk factor for increased postsurgical mortality; very good results have been reported [17-19].

In our thought minimally invasive stand-alone surgical ablation should represent the next therapeutic step following one or more unsuccessful catheter ablations. The benefits of this procedure are direct visualization using minithoracotomies, absence of fluoroscopy, and possibility to exclude the left atrial appendage and to address multiple potential mechanism of atrial fibrillation (ectopic foci, ligament of Marshall, and autonomic ganglia). Thus with this approach, it is possible to perform continuous and transmural lesions that should be more difficult to recover in respect to the endocardial ones.

Our data are noteworthy since they show the success rate of this procedure in a population of consecutive patients with atrial fibrillation, $73 \%$ of whom had not responded to previous multiple catheter pulmonary vein ablations. Since not all these previous endocardial procedures were performed in our center, it is difficult to understand the possible causes of these failures. We hypothesize that the failure of previous endocardial approaches was due to conduction recovery in some connection points between PVs and the atrium.

Our 12 -month $73 \%$ success rate (defined as freedom from any atrial arrhythmia recurrence longer than $30 \mathrm{~s}$ and 
from antiarrhythmic therapy) is based on strict ECG home monitoring on a daily basis during the first 4 months and later on quarterly $24-\mathrm{h}$ Holter monitoring. This was considered enough in the last years [15], but it has been demonstrated by many studies that the detection of atrial fibrillation increases on prolonging the monitoring time [20]. For this reason, strict ECG monitoring is fundamental to ensuring that asymptomatic arrhythmia recurrences are detected. According to the new guidelines [16], a monthly 24-h Holter monitoring or a daily ECG monitoring should be performed for at least 1 year of follow-up. Currently we follow these recommendations in implanting a continuous cardiac monitor in all the patients undergoing this procedure, as performed in the last three patients enrolled in this cohort.

Although our population included higher proportions of patients with persistent atrial fibrillation, previous failed catheter ablations, and daily ECG monitoring, our results are comparable, in terms of freedom from any atrial arrhythmia, to those reported in previous studies with even less strict success definitions $[9,13,15]$. We decided not to consider the first 3 months as a blanking period because we have noted that early recurrences are predictive of later recurrences in the follow-up period, as already reported by other authors [14].

We found an $18 \%$ incidence of new-onset left atrial flutter in patients that previously performed catheter ablation. This very high incidence of new-onset atrial flutter could be due to the fact that no extra lines apart from pulmonary vein isolations were performed in these patients both during catheter and surgical ablation. Anyway, due to the fact that the average time of first atrial flutter recurrence is $8 \pm 5$ months following surgical ablation, it is not possible to verify if there is a direct correlation with the surgical procedure or with the catheter ablation.

It is important to underline that previously ineffective antiarrhythmic drugs, even after catheter ablation, started to control atrial fibrillation after the surgical procedure in the majority of patients. Probably this is due to the substrate modification induced by the surgical procedure. The efficacy of the procedure is maintained even after 2 years of follow-up without the addictive effect of antiarrhythmic therapy.

Autonomic denervation and ligament of Marshall resection have been suggested to eliminate the triggering of pulmonary vein firing from the left atrium, improving the outcome of the procedure $[10,12,14]$. However, it is not yet demonstrated whether there is an additive effect of autonomic ganglia ablation in terms of success rate, in that discrepant results have emerged from previous studies [14]. Also in our population, it is not possible to draw conclusion, since these procedures were performed in all the patients. Atrial appendage exclusion also offers benefits in terms of stroke prevention, though further studies on this 
issue are needed in order to ascertain whether it is safe to discontinue oral anticoagulant therapy in these patients [21].

We think that, in some cases, a hybrid approach (catheter plus surgical ablation) could yield a higher success rate. In our population, previous catheter ablation was not predictive of greater success even if success rate in this subgroup of patients could be attributed to the combination of the two treatments. However, this may have been due to the limited number of patients.

\section{Limitations}

Our experience is on a limited number of patients with different types of atrial fibrillation undergoing minimally invasive surgical ablation of atrial fibrillation. As for previous studies on this topic, results cannot be extended to other centers performing the same procedure. Moreover, in the majority of patients, the intensity of monitoring was higher during the period of pharmacological support and lower when antiarrhythmic therapy was stopped, thus introducing a potential bias in the results. More investigations, including multicenter randomized studies, are required in order to determine whether the risk/benefit ratio and the costs of this new technique are acceptable.

\section{Conclusions}

Minimally invasive surgical ablation in patients with atrial fibrillation is a feasible technique and gave satisfactory results that persist at long-term follow-up. Atrial fibrillation recurrence alone is low, while we still have a high incidence of atrial flutter and tachycardia in the follow-up. It remains to be proven that atrial flutter recurrences can be managed by subsequent flutter ablation. Further multicenter studies involving larger populations are needed in order to confirm the results of single-center experiences and to update atrial fibrillation guidelines on this procedure.

Conflict of interest No conflict of interest exists.

Open Access This article is distributed under the terms of the Creative Commons Attribution Noncommercial License which permits any noncommercial use, distribution, and reproduction in any medium, provided the original author(s) and source are credited.

\section{References}

1. Feinberg, W. M., Blackshear, J. L., Laupacis, A., Kronmal, R., \& Hart, R. G. (1995). Prevalence, age distribution, and gender of patients with atrial fibrillation. Analysis and implications. Archives of Internal Medicine, 155(5), 469-473.

2. Krahn, A. D., Manfreda, J., Tate, R. B., Mathewson, F. A., \& Cuddy, T. E. (1995). The natural history of atrial fibrillation: incidence, risk factors, and prognosis in the Manitoba Follow-Up Study. The American Journal of Medicine, 98, 476-484.

3. Wazni, O. M., Marrouche, N. F., Martin, D. O., Verma, A., Bhargava, M., Saliba, W., et al. (2005). Radiofrequency ablation vs antiarrhythmic drugs as first-line treatment of symptomatic atrial fibrillation: a randomized trial. JAMA, 293 (21), 2634-2640.

4. Hindricks, G., Piorkowski, C., Tanner, H., Kobza, R., Gerds-Li, J. H., Carbucicchio, C., et al. (2005). Perception of atrial fibrillation before and after radiofrequency catheter ablation: relevance of asymptomatic arrhythmia recurrence. Circulation, 112(3), 307313.

5. Piorkowski, C., Kottkamp, H., Tanner, H., Kobza, R., Nielsen, J. C., Arya, A., et al. (2005). Value of different follow-up strategies to assess the efficacy of circumferential pulmonary vein ablation for the curative treatment of atrial fibrillation. Journal of Cardiovascular Electrophysiology, 16(12), 1286-1292.

6. Oral, H., Chugh, A., Lemola, K., Cheung, P., Hall, B., Good, E., et al. (2004). Noninducibility of atrial fibrillation as an end point of left atrial circumferential ablation for paroxysmal atrial fibrillation: a randomized study. Circulation, 110(18), 2797-2801.

7. Cappato, R., Calkins, H., Chen, S. A., Davies, W., Iesaka, Y., Kalman, J., et al. (2010). Updated worldwide survey on the methods, efficacy and safety of catheter ablation for human atrial fibrillation. Circulation: Arrhythmia and Electrophysiology, 3(1), 32-38.

8. Edgerton, J. R., McClelland, J. H., Duke, D., Gerdisch, M. W., Steinberg, B. M., Bronleewe, S. H., et al. (2009). Minimally invasive surgical ablation of atrial fibrillation: six-month results. The Journal of Thoracic and Cardiovascular Surgery, 138, 109114.

9. Beyer, E., Lee, R., \& Lam, B. K. (2009). Point: Minimally invasive bipolar radiofrequency ablation of lone atrial fibrillation: early multicenter results. The Journal of Thoracic and Cardiovascular Surgery, 137, 521-526.

10. Bagge, L., Blomström, P., Nilsson, L., Einarsson, G. M., Jidéus, L., \& Blomström-Lundqvist, C. (2009). Epicardial off-pump pulmonary vein isolation and vagal denervation improve long-term outcome and quality of life in patients with atrial fibrillation. The Journal of Thoracic and Cardiovascular Surgery, 137(5), 1265-1271.

11. Bauer, M., \& Hetzer, R. (2009). Video-assisted thoracoscopic surgical treatment of lone atrial fibrillation. MMCTS. European Association for Cardio-thoracic Surgery. Available at: http://mmcts. ctsnetjournals.org. Accessed 27 Nov 2009.

12. Edgerton, J. R., Edgerton, Z. J., Weaver, T., Reed, K., Prince, S., Herbert, M. A., et al. (2008). Minimally invasive pulmonary vein isolation and partial autonomic denervation for surgical treatment of atrial fibrillation. The Annals of Thoracic Surgery, 86, 35-39.

13. Klinkenberg, T. J., Ahmed, S., Ten Hagen, A., Wiesfeld, A. C., Tan, E. S., Zijlstra, F., et al. (2009). Feasibility and outcome of epicardial pulmonary vein isolation for lone atrial fibrillation using minimal invasive surgery and high intensity focused ultrasound. Europace, 11 (12), 1624-1631.

14. Han, F. T., Kasirajan, V., Kowalski, M., Kiser, R., Wolfe, L., Kalahasty, G., et al. (2009). Results of minimally invasive surgical pulmonary vein isolation and ganglionic plexi ablation for atrial fibrillation. Circulation: Arrhythmia and Electrophysiology, 2(4), 370-377.

15. Calkins, H., Brugada, J., Packer, D. L., Cappato, R., Chen, S. A., Crijns, H. J., et al. (2007). HRS/EHRA/ECAS expert consensus statement on catheter and surgical ablation of atrial fibrillation: 
recommendations for personnel, policy, procedures and follow-up. Europace, 9, 335-379.

16. European Heart Rhythm Association, European Association for Cardio-Thoracic Surgery, Camm, A. J., Kirchhof, P., Lip, G. Y., Schotten, U., et al. (2010). Guidelines for the management of atrial fibrillation: the Task Force for the Management of Atrial Fibrillation of the European Society of Cardiology (ESC). European Heart Journal, 31(19), 23692429.

17. Polymeropoulos, K. P., Rodriguez, L. M., Timmermans, C., \& Wellens, H. J. (2002). Images in cardiovascular medicine. Radiofrequency ablation of a focal atrial tachycardia originating from the Marshall ligament as a trigger for atrial fibrillation. Circulation, 105(17), 2112-2113.
18. Benussi, S., Galanti, A., Nascimbene, S., Fumero, A., Dorigo, E., Zerbi, V., et al. (2009). Complete right atrial ablation with bipolar radiofrequency. The Annals of Thoracic Surgery, 87(5), 1573-1576.

19. Filardo, G., Hamilton, C., Hebeler, R. F., Jr., Hamman, B., \& Grayburn, P. (2009). New-onset postoperative atrial fibrillation after isolated coronary artery bypass graft surgery and long-term survival. Circulation: Cardiovascular Quality and Outcomes, 2(3), 164-169.

20. Arya, A., Piorkowski, C., Sommer, P., Kottkamp, H., \& Hindricks, G. (2007). Clinical implications of various follow up strategies after catheter ablation of atrial fibrillation. Pacing and Clinical Electrophysiology, 30(4), 458-462.

21. Blackshear, J. L., \& Odell, J. A. (1996). Appendage obliteration to reduce stroke in cardiac surgical patients with atrial fibrillation. The Annals of Thoracic Surgery, 61(2), 755-759. 